\title{
Rational Design for Optimizing Hybrid Thermo-triboelectric Generators Targeting Human Activities
}

\author{
Byungseok Seo ${ }^{\dagger, \S}$, Youngsun $\mathrm{Cha}^{\dagger, \S}$, Sangtae Kim ${ }^{*}$, , and Wonjoon Choi ${ }^{*}$, \\ $\dagger$ School of Mechanical Engineering, Korea University, Seoul, Korea, 136-701 \\ Center for Electronic Materials, Korea Institute of Science and Technology, Seoul, Korea
}

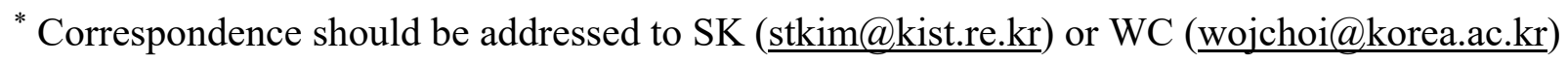

$\S$ These authors contributed equally to this work. 


\section{Experimental Methods}

\section{Fabrication of HThTG comprising of PDMS and Bi $\mathrm{Te}_{3}$ tiles}

A polyethylene terephthalate (PET) film (20 $\mu \mathrm{m}$ in thickness) was prepared as a substrate for the HThTG. One triboelectric electrode was prepared by attaching aluminum tape on the substrate. The prepared PET film was cleaned using deionized water and a nitrogen blow gun. PDMS (Sylgard 184, Dow corning) and curing agent were mixed using a vortex machine at the mass ratio of 10:1 for $60 \mathrm{~s}$. The mixed PDMS $(8.5 \mathrm{~g})$ was poured and distributed using the spin coating method at $100 \mathrm{rpm}$ for $45 \mathrm{~s}$ on the PET and cured at $80{ }^{\circ} \mathrm{C}$ for $2 \mathrm{~h}$. Shadow masks ( $5 \mathrm{~mm}$ in thickness) with $5 \times 5$ square array patterns on acryl plates were fabricated using a laser cutter. $\mathrm{Bi}_{2} \mathrm{Te}_{3}$ tiles $(5 \mathrm{~mm} \times 5 \mathrm{~mm} \times 2 \mathrm{~mm})$ were arranged in a square pattern. Upper sides of the $\mathrm{Bi}_{2} \mathrm{Te}_{3}$ tiles were connected to each other via aluminum tape and silver epoxy paste. The silver epoxy paste was dried at room temperature for 24 h. The tiles and aluminum tape were then bound to the PDMS substrates using non-cured PDMS as glue. Subsequently, the shadow masks were peeled off from the HThTG, and PDMS was poured into the empty space in between the tiles up to the height of the $\mathrm{Bi}_{2} \mathrm{Te}_{3}$ tiles. The step-by-step fabrication process is illustrated in Figure S1.

\section{Characterization of material properties employed in the HThTG}

The Seebeck coefficients of $\mathrm{Bi}_{2} \mathrm{Te}_{3}$ were characterized by a four-point probe station. The timedependent heat equation and Seebeck effect were used to calculate the characteristic time constants, which related the generated thermoelectric voltage and temperature difference. To minimize the error from contacts, four $\mathrm{Bi}_{2} \mathrm{Te}_{3}$ pellets were connected in series, and total measurements were obtained for $\mathrm{p} / \mathrm{n}$ type devices. The electrical resistance of serially connected $\mathrm{Bi}_{2} \mathrm{Te}_{3}$ was measured using a digital multimeter (Fluke 1557). The surface morphology of $\mathrm{Bi}_{2} \mathrm{Te}_{3}$ pellets was obtained using scanning electron microscopes (FEI, Model Quanta 250 FEG and Jeol, Model JSM-6701 F). 


\section{Measurement of thermoelectric output}

The measurements of thermoelectric output were carried out under the two end-point temperatures of 27 and $34.5{ }^{\circ} \mathrm{C}$. The output voltage was measured by a digital phosphor oscilloscope (Tektronix DPO2004B), and the SCC was measured using Agilent 34464A with a Number of Power Line Cycles (NPLC) of 0.02. The temporal evolution of temperature was analyzed in detail and fitted to exponential curves.

\section{Measurement of triboelectric output}

Triboelectric output was obtained using a homemade touch machine based on DC motors (Figure S18). The touch arm was covered with nylon considering its similar electron affinity to that of human skin. The specific frequency of the triboelectric contact was controlled by the DC motor. The OCV and voltage drop across varied external loads $\left(10\right.$ to $\left.10^{8} \Omega\right)$ were measured using digital phosphor oscilloscopes (Tektronix DPO2004B). The SCC was obtained using Agilent 34464A digital multimeter with an NPLC of 0.02 .

\section{Simulation of transient thermal distribution in the thermoelectric part of the HThTGs}

Transient temperature distribution within the thermoelectric generator was modeled using finite element method conducted through the COMSOL Multiphysics simulation package. Time-dependent thermal constants were calculated based on the detailed heat transfer analyses within the bulk $\mathrm{Bi}_{2} \mathrm{Te}_{3}$ tiles and the interfaces between the tiles and PDMS layers. The initial parameters employed in the simulation involved convection coefficient $\left(10 \mathrm{~W} / \mathrm{m}^{2} \mathrm{k}\right)$, ambient temperature $(298.13 \mathrm{~K})$, and human skin temperature $(308.13 \mathrm{~K})$. The thermal constants were estimated from the average temperature of the tile surfaces. The Seebeck coefficients were assumed constant for temperature differences below $10 \mathrm{~K}$. 
Table S1. PDMS surface area and volume according to tile spacing.

\begin{tabular}{|c|c|c|c|c|}
\hline $\begin{array}{c}\text { Tile spacing } \\
(\mathrm{mm})\end{array}$ & $\begin{array}{c}\text { PDMS } \\
\text { surface area }\left(\mathrm{cm}^{2}\right)\end{array}$ & $\begin{array}{c}\text { PDMS } \\
\text { volume }\left(\mathrm{cm}^{3}\right)\end{array}$ & $\begin{array}{c}\mathrm{Bi}_{2} \mathrm{Te}_{3} \\
\text { volume }\left(\mathrm{cm}^{3}\right)\end{array}$ & $\begin{array}{c}\text { Total device } \\
\text { volume }\left(\mathrm{cm}^{3}\right)\end{array}$ \\
\hline 0.5 & 1.04 & 0.22 & 1.3125 & 1.5309 \\
\hline 1 & 2.16 & 0.45 & 1.3125 & 1.7661 \\
\hline 1.5 & 3.36 & 0.71 & 1.3125 & 2.0181 \\
\hline 2 & 4.64 & 0.97 & 1.3125 & 2.2869 \\
\hline 2.5 & 6 & 1.26 & 1.3125 & 2.5725 \\
\hline 3 & 7.44 & 1.56 & 1.3125 & 2.8749 \\
\hline 3.5 & 8.96 & 1.88 & 1.3125 & 3.1941 \\
\hline 4 & 10.56 & 2.22 & 1.3125 & 3.5301 \\
\hline 4.5 & 12.24 & 2.57 & 1.3125 & 3.8829 \\
\hline
\end{tabular}




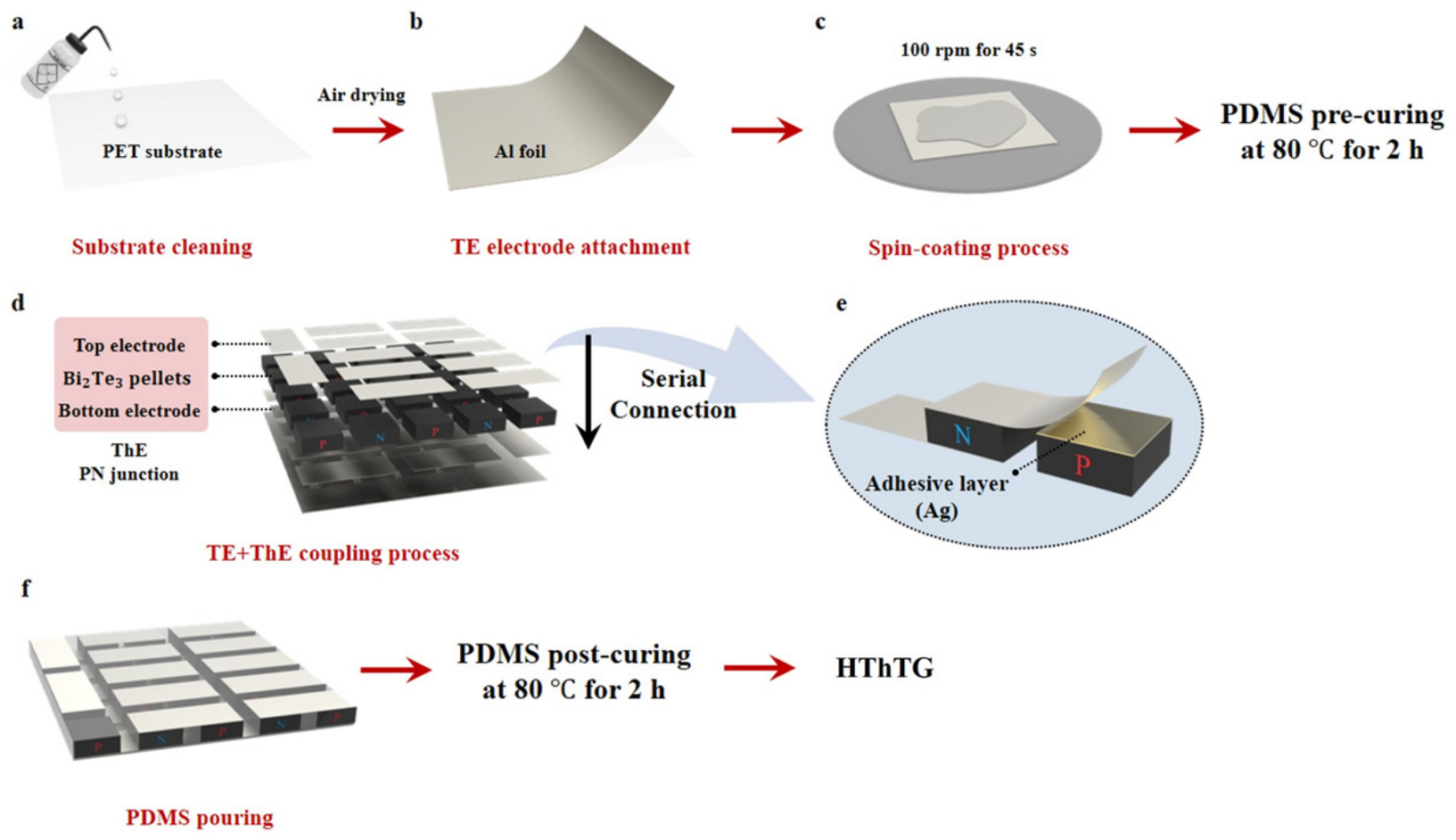

Figure S1. Fabrication process of the HThTG. (a) Surface cleaning process of PET substrate with acetone and deionized water. (b) Attachment of aluminum foil on the substrate for the triboelectric (TE) electrode layer. (c) PDMS deposition via spin coating to separate electrode layers between the thermoelectric (ThE) and TE parts, and the subsequent pre-curing process at $80{ }^{\circ} \mathrm{C}$ for $2 \mathrm{~h}$. (d and e) Arrangement of $\mathrm{Bi}_{2} \mathrm{Te}_{3}$ tiles into the pn junction and serial electrode installation via silver adhesive layers. (f) PDMS filling of the remaining spaces in between the $\mathrm{Bi}_{2} \mathrm{Te}_{3}$ tiles and the post curing process at $80{ }^{\circ} \mathrm{C}$ for $2 \mathrm{~h}$. 
$\mathbf{a}$

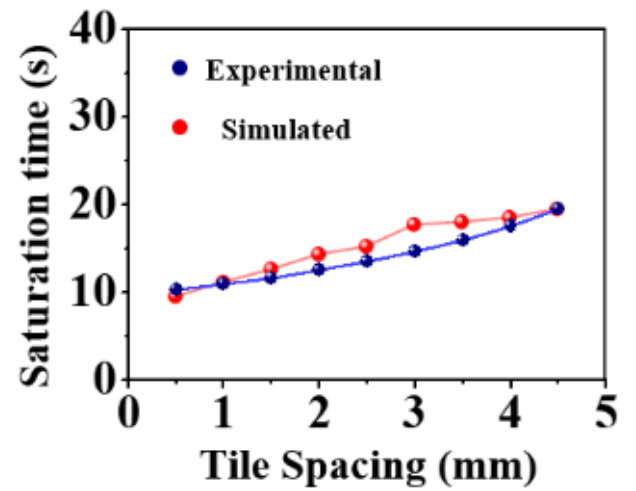

c

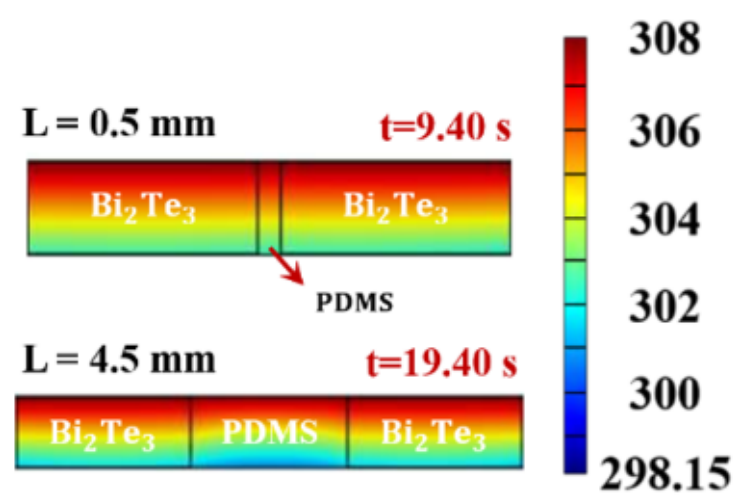

b

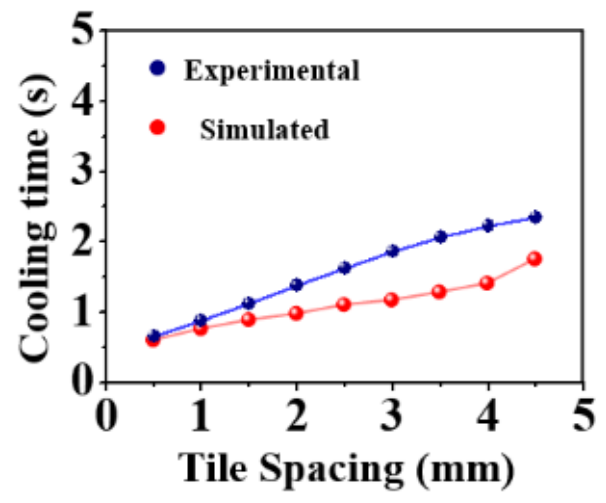

d 302

301

300

299

298.15

Figure S2. Modeled heat transport phenomena within the HThTG and its comparison with the experimental results. Time taken to achieve $63.2 \%$ of thermal saturation in the (a) saturation and (b) cooling regions according to the tile spacing. The simulated cross-sectional view of the device when $63.2 \%$ of (c) thermal saturation and (d) cooling occurred. 

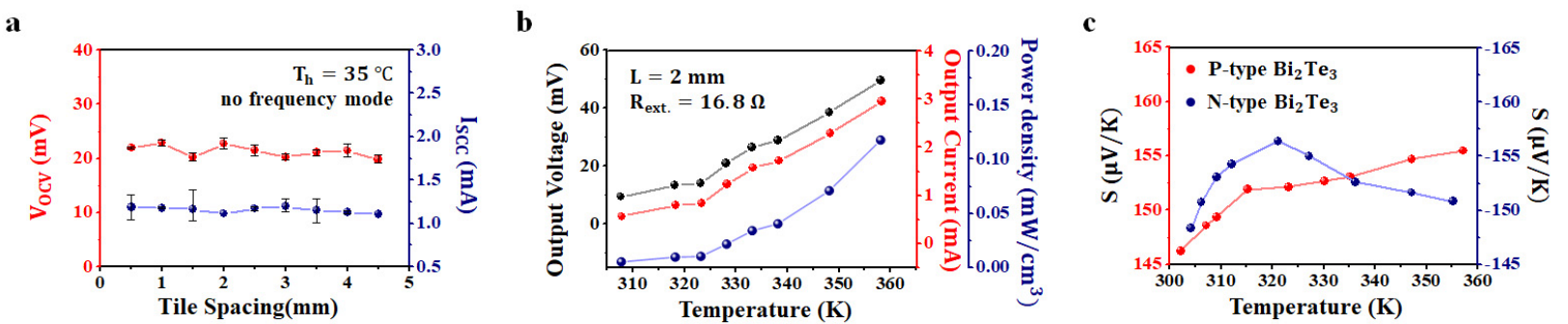

Figure S3. Electrical characteristics of the thermoelectric part of the HThTG. (a) OCV and SCC measurement with distinct tile spacings when the device was continuously in contact with human skin. (b) Performance evaluation of output power generation upon temperature change of contact surface with $16.8 \Omega$ of external load. (c) Temperature dependent Seebeck coefficient (S) measurement of each p-type and n-type bismuth telluride tile. 

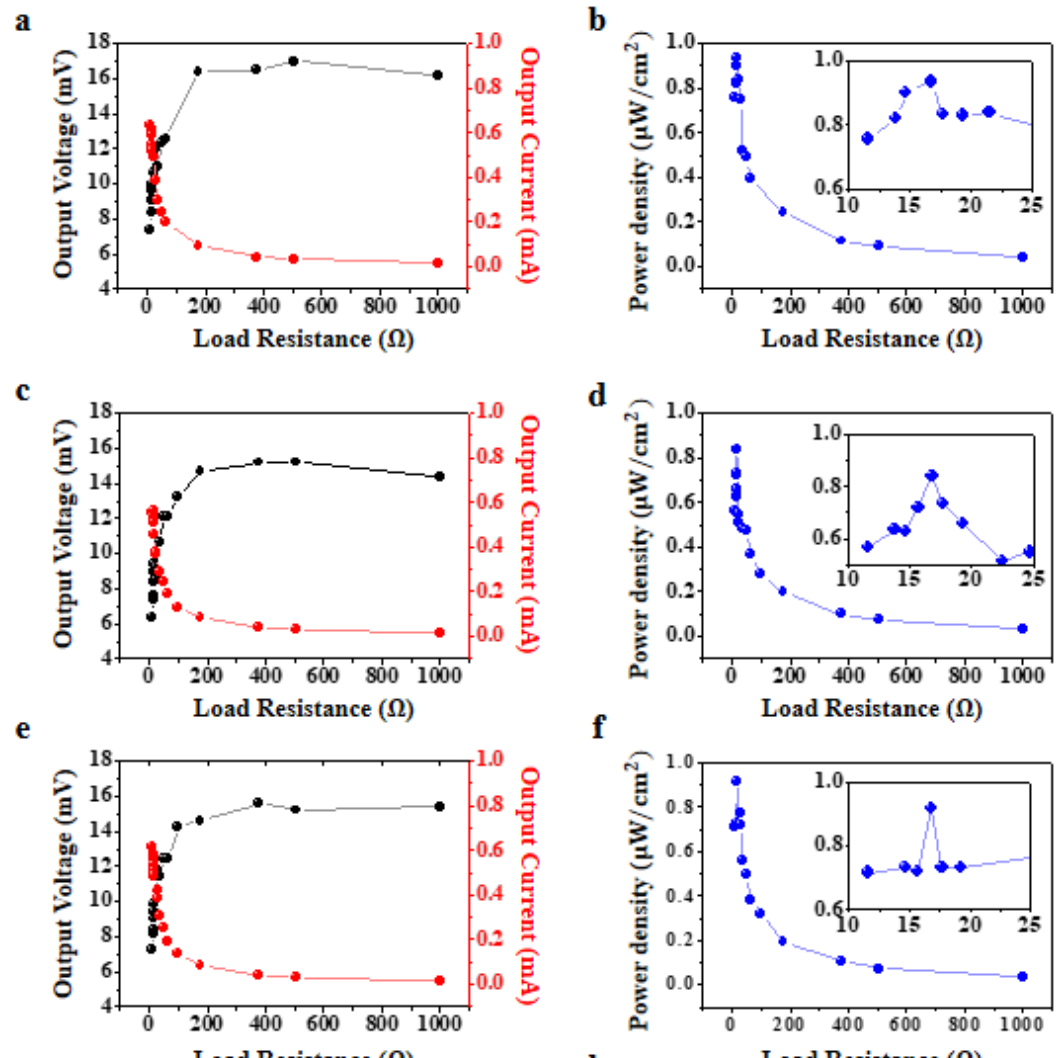

f
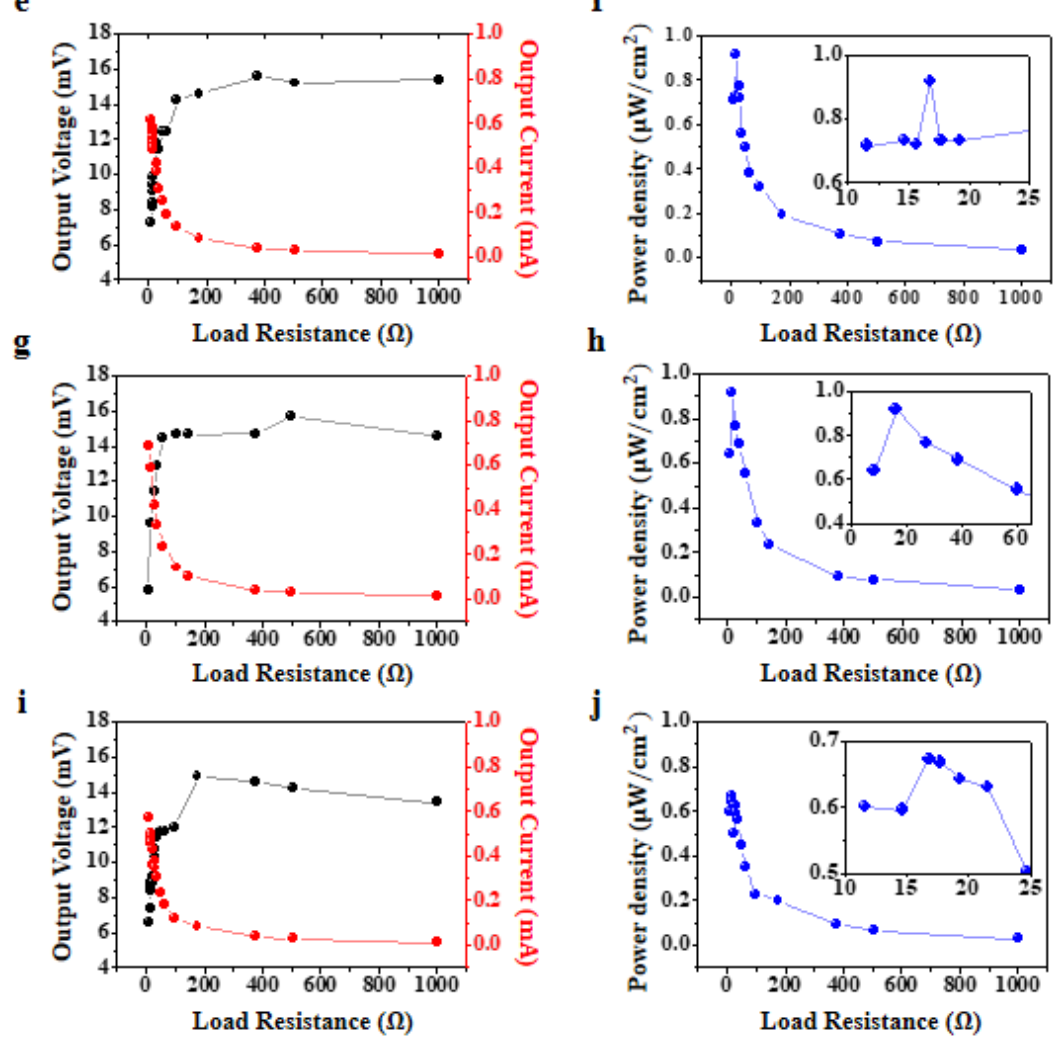

Figure S4. Impedance matching of the thermoelectric part in the HThTG. The output voltage across varied resistors and the output current and power density are measured for tile spacings of (a and b) 1, (c and d) 2.5, (e and f) 3, (g and h) 4, and (i and j) $4.5 \mathrm{~mm}$. 
a

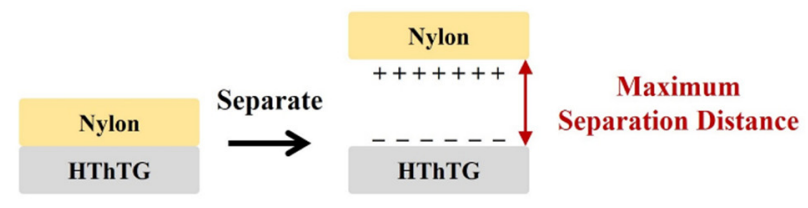

Contact moment

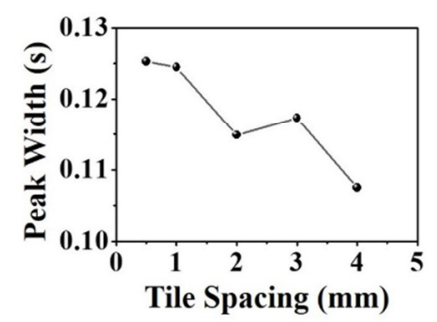

b

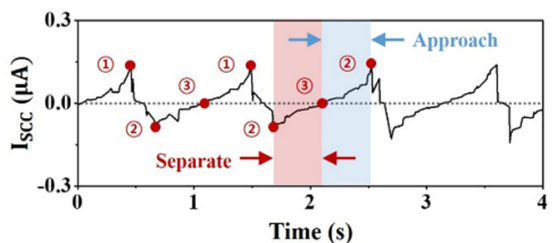

(1) Contact moment

(2) Separate moment

(3) At maximum separation distance

d

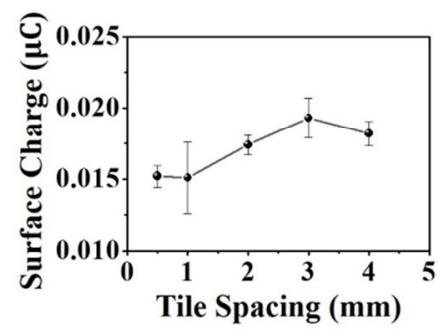

Figure S5. ( $a$ and b) The schematic representation and SCC plot showing the generation of triboelectric current upon touch and separation. (c) The peak SCC width decreases with increasing tile spacing values. (d) The amount of generated triboelectric charges according to tile spacing. 


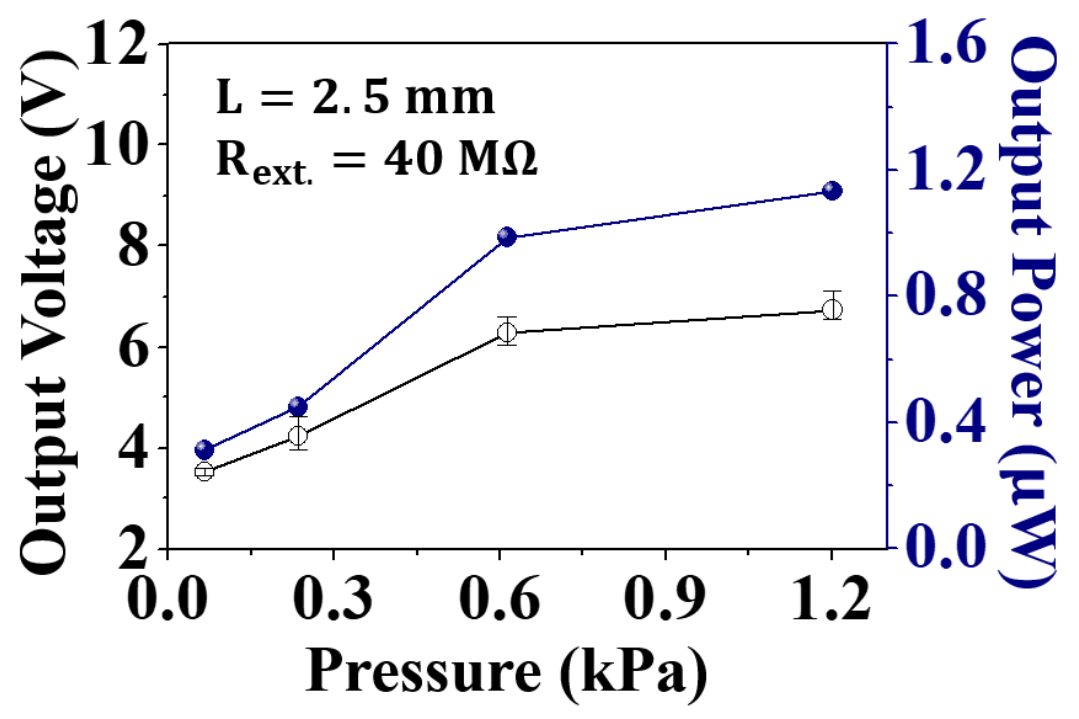

Figure S6. Triboelectric voltage and power obtained at varied mechanical input load. A tile spacing of $2.5 \mathrm{~mm}$ was used with the external load of $40 \mathrm{M} \Omega$ at $1 \mathrm{~Hz}$ frequency. 

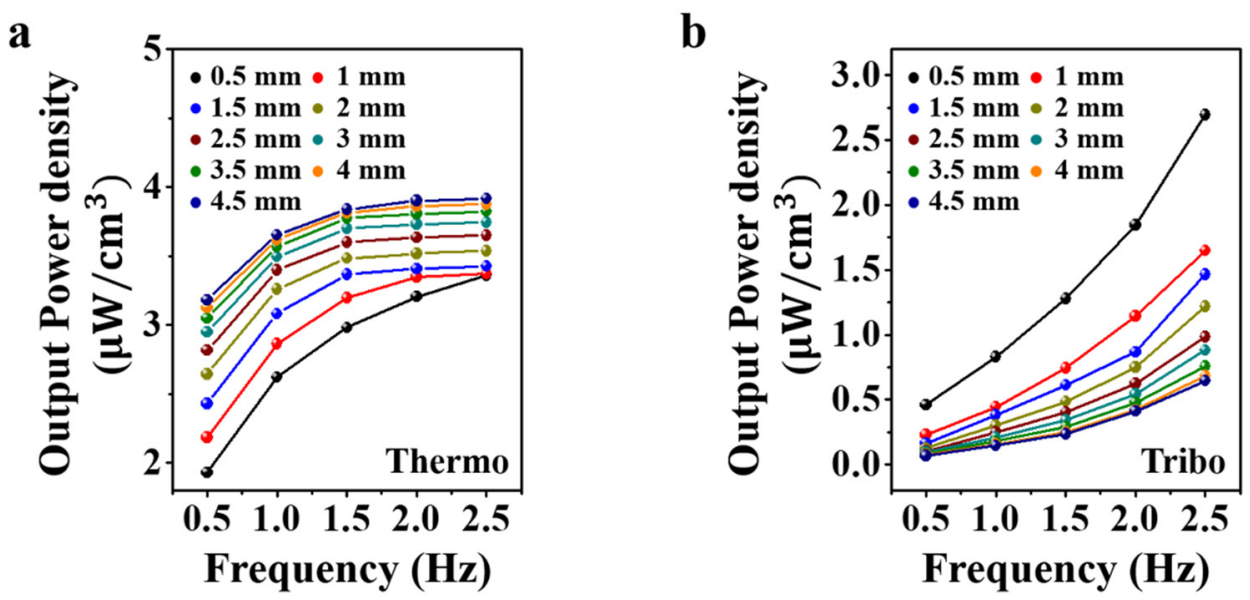

Figure S7. Power densities of (a) thermoelectric and (b) triboelectric generators when the generated powers from each mode were divided by the area of thermoelectric and triboelectric materials $\left(\mathrm{Bi}_{2} \mathrm{Te}_{3}\right.$ and PDMS, respectively). 
a

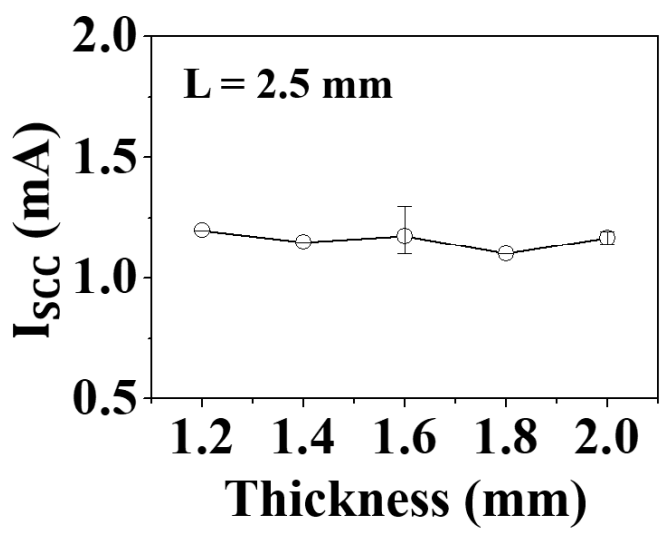

b

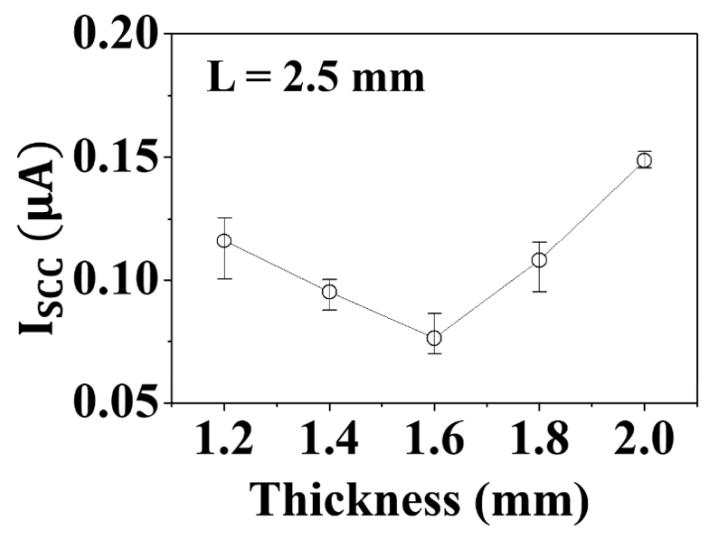

Figure S8. (a) The peak current outputs of thermoelectrics, obtained at five distinct $\mathrm{Bi}_{2} \mathrm{Te}_{3}$ pellet thicknesses. (b) The peak current outputs of triboelectrics, obtained at five distinct PDMS layer thicknesses.

In the case of thermoelectrics, the generated electric current scales inversely with the thickness of the thermoelectrics and scales with the area of the thermoelectrics, similar to a typical resistor. Thus, we expected that the thermoelectric power generation would decrease with increased $\mathrm{Bi}_{2} \mathrm{Te}_{3}$ pellet thickness. The figure below shows that the maximum thermoelectric current output remains more or less identical regardless of the pellet thicknesses. We note that the increased pellet thickness increases the overall thermal resistance while at the same time increasing the overall resistance of the device. The increased thermal resistance enhances the electric current, yet the increased resistance cancels out the current enhancement, resulting in the similar peak thermoelectric current among all $\mathrm{Bi}_{2} \mathrm{Te}_{3}$ and PDMS thicknesses.

In the case of triboelectric generators, various literature reports that the thickness of triboelectric material (the negative-charging material) does not significantly affect the current generation, especially when the thickness lies in the range above $100 \mathrm{~nm}$ [HE, Xianming, et al., Nanoscale, 2015, 7.5, 1896-1903 and CUI, Nuanyang et al., ACS Nano, 2016, 10.6, 6131-6138]. Specifically, the charge generation does not get affected significantly by PDMS thickness, unless the thickness becomes thin enough that the PDMS roughness follows that of the substrates. Figure S8 also shows no conclusive trend between the thickness of PDMS and peak current output. 


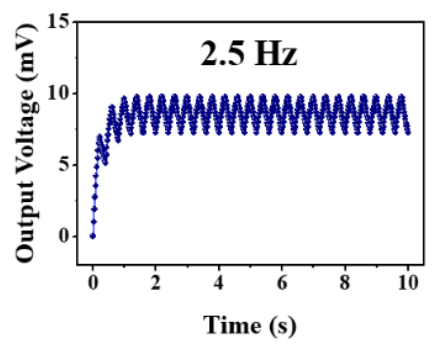

d

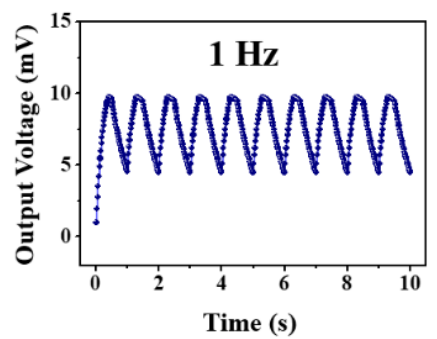

b

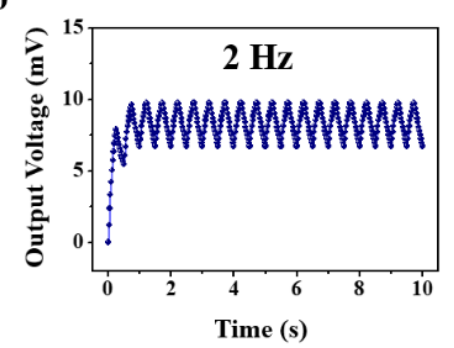

e

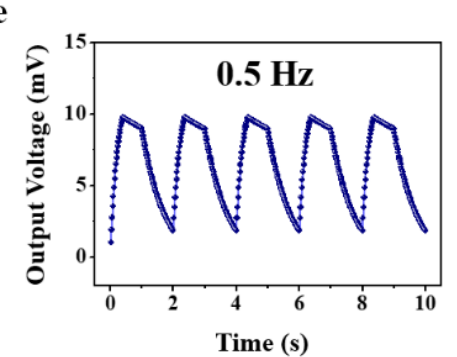

c

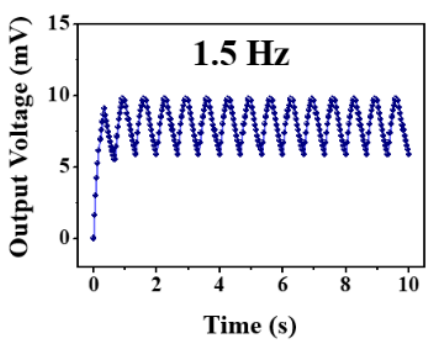

f

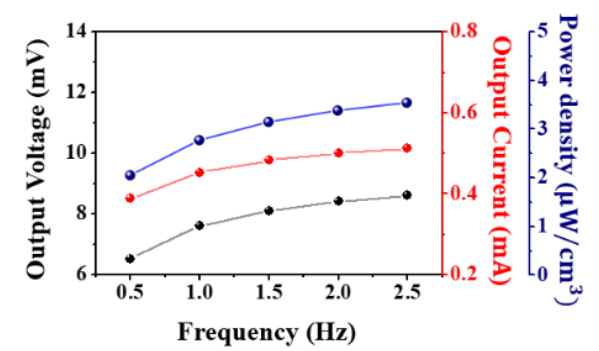

Figure S9. Output voltage signals for the HThTG with a tile spacing of $0.5 \mathrm{~mm}$ when human touch temperature is repeatedly applied at (a) 2.5, (b) 2, (c) 1.5 , (d) 1, and (e) $0.5 \mathrm{~Hz}$ at an external load of $16.8 \Omega$. (f) The analysis of output voltage, current, and power density according to frequency change. 


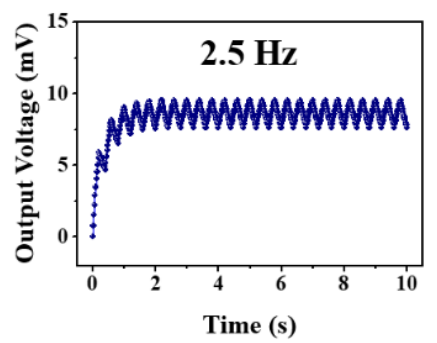

d

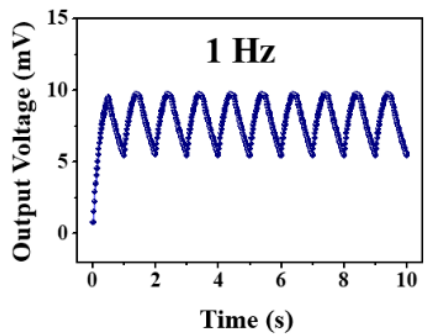

b

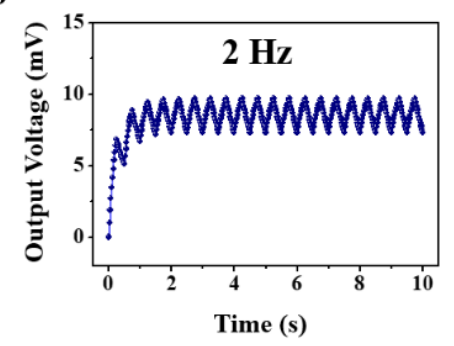

e

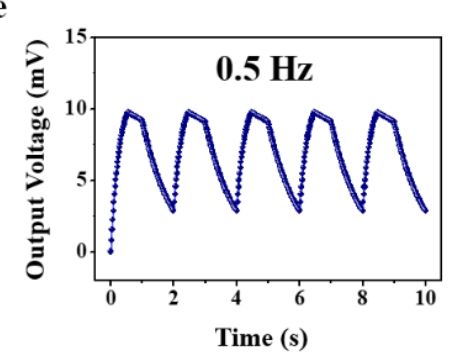

c

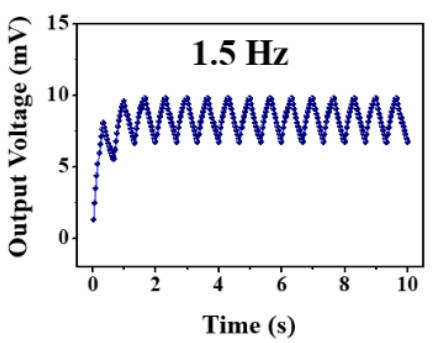

f

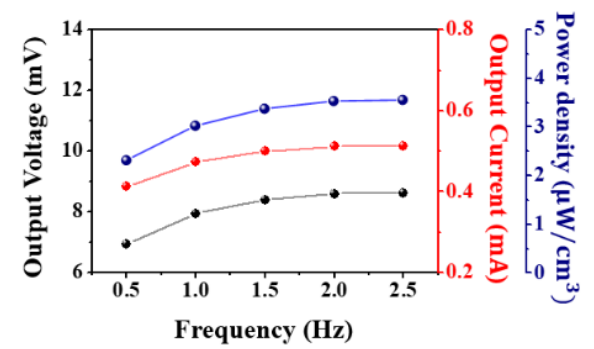

Figure S10. Output voltage signals for the HThTG with a tile spacing of $1 \mathrm{~mm}$ when human touch temperature is repeatedly applied at (a) 2.5 , (b) 2, (c) 1.5 , (d) 1 , and (e) $0.5 \mathrm{~Hz}$ at an external load of $16.8 \Omega$. (f) The analysis of output voltage, current, and power density according to frequency change. 
a

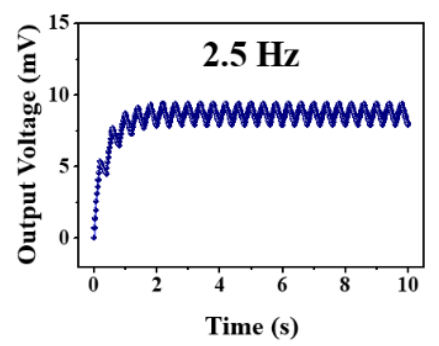

d

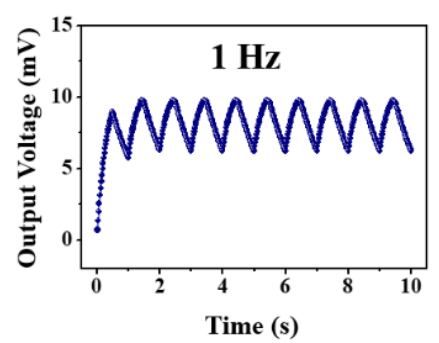

b

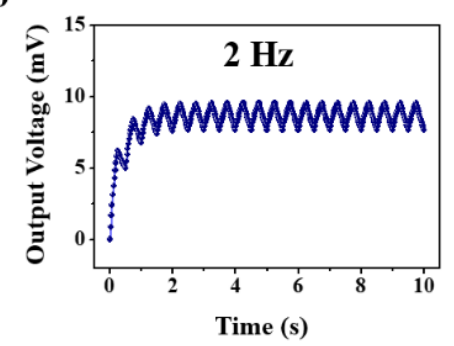

e

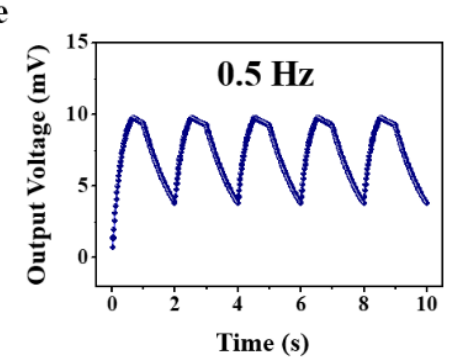

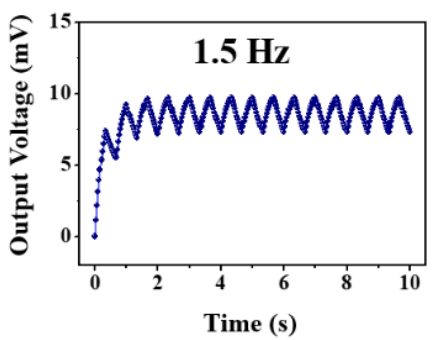

f

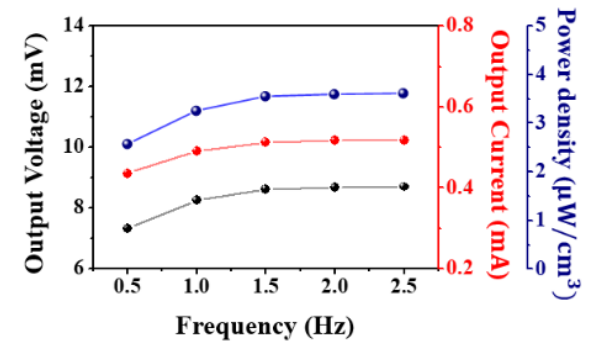

Figure S11. Output voltage signals for the HThTG with a tile spacing of $1.5 \mathrm{~mm}$ when human touch temperature is repeatedly applied at (a) 2.5, (b) 2, (c) 1.5 , (d) 1 , and (e) $0.5 \mathrm{~Hz}$ at an external load of $16.8 \Omega$. (f) The analysis of output voltage, current, and power density according to frequency change. 


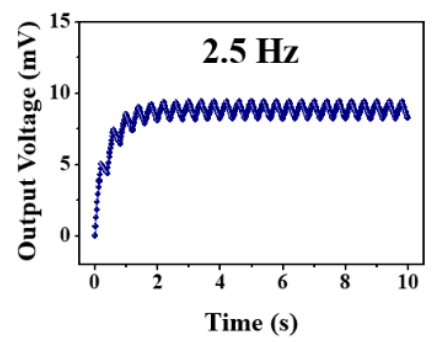

d

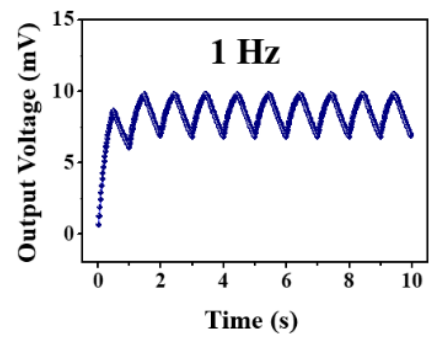

b

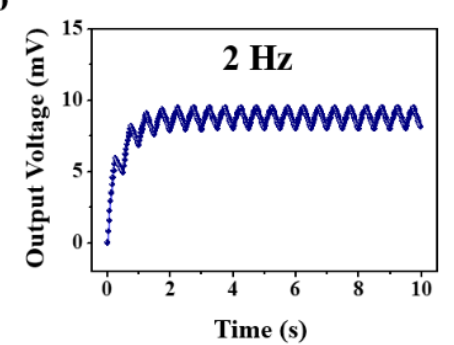

e

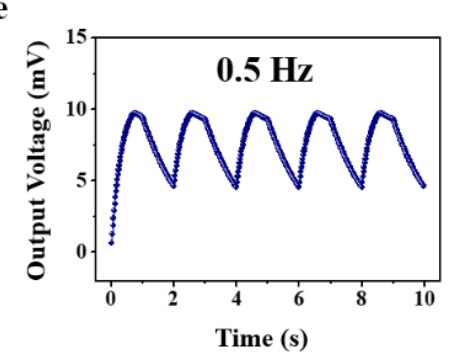

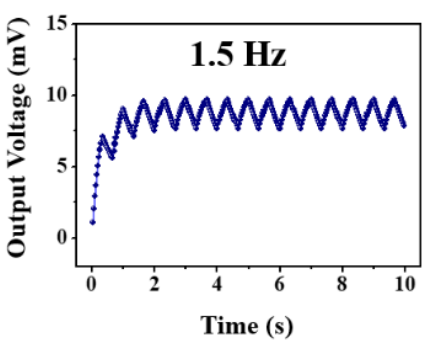

f

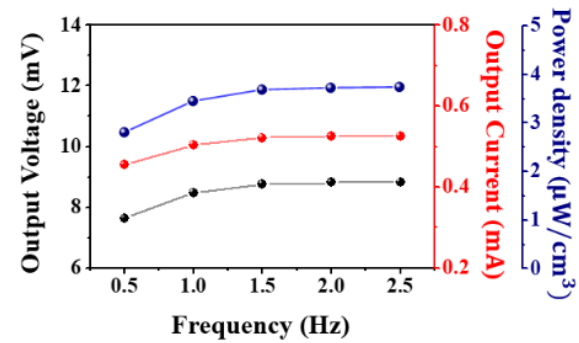

Figure S12. Output voltage signals for the HThTG with a tile spacing of $2 \mathbf{~ m m}$ when human touch temperature is repeatedly applied at (a) 2.5 , (b) 2, (c) 1.5 , (d) 1 , and (e) $0.5 \mathrm{~Hz}$ at an external load of $16.8 \Omega$. (f) The analysis of output voltage, current, and power density according to frequency change. 


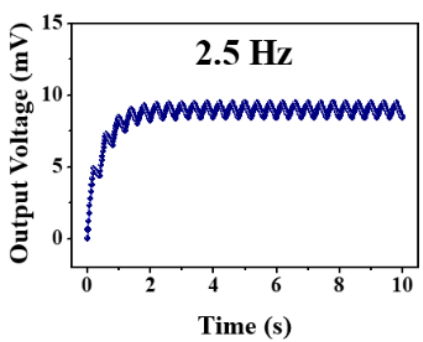

d

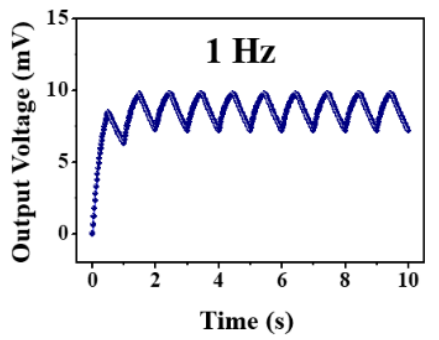

b

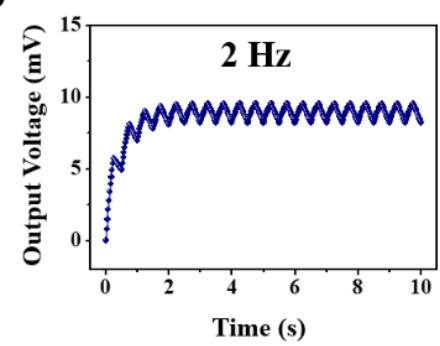

e

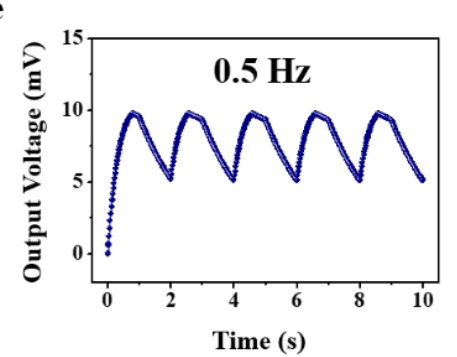

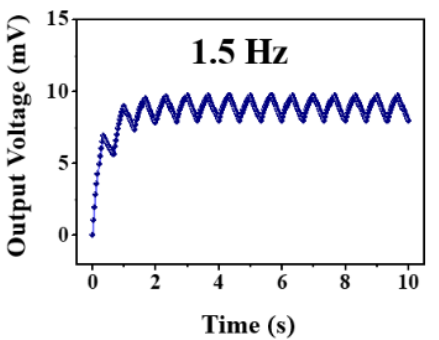

f

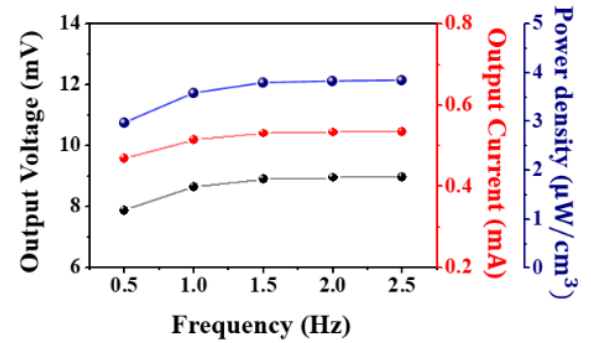

Figure S13. Output voltage signals for the HThTG with a tile spacing of $2.5 \mathrm{~mm}$ when human touch temperature is repeatedly applied at (a) 2.5 , (b) 2 , (c) 1.5 , (d) 1 , and (e) $0.5 \mathrm{~Hz}$ at an external load of $16.8 \Omega$. (f) The analysis of output voltage, current, and power density according to frequency change. 


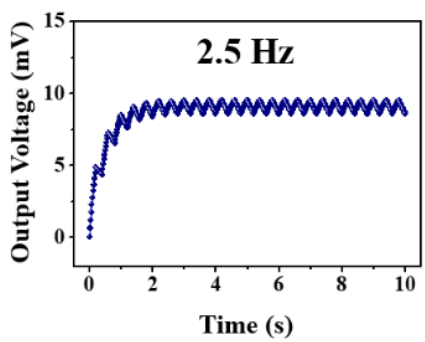

d

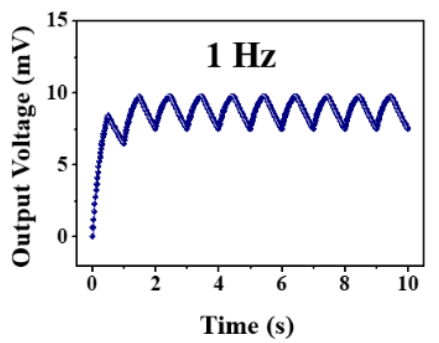

b

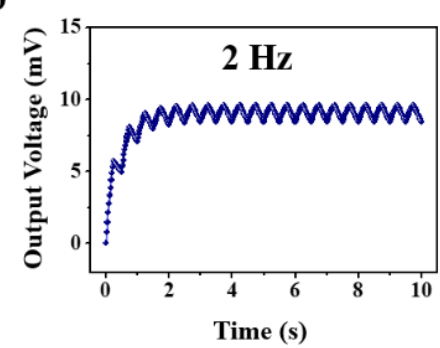

e

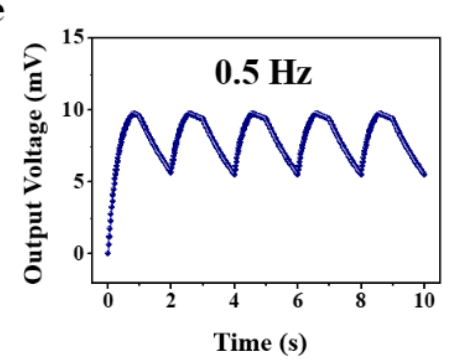

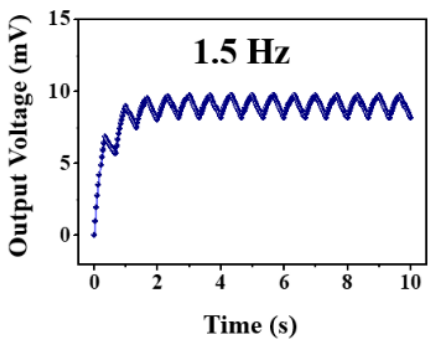

$\mathbf{f}$

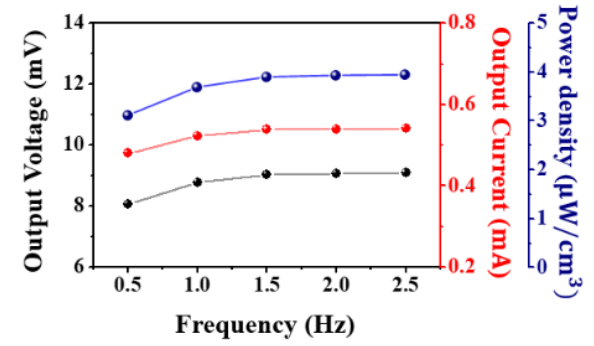

Figure S14. Output voltage signals for the HThTG with a tile spacing of $3 \mathbf{~ m m}$ when human touch temperature is repeatedly applied at (a) 2.5 , (b) 2 , (c) 1.5 , (d) 1 , and (e) $0.5 \mathrm{~Hz}$ at an external load of $16.8 \Omega$. (f) The analysis of output voltage, current, and power density according to frequency change. 


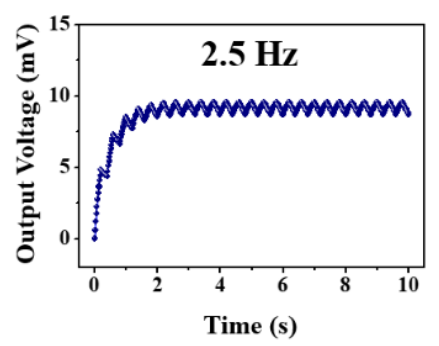

d

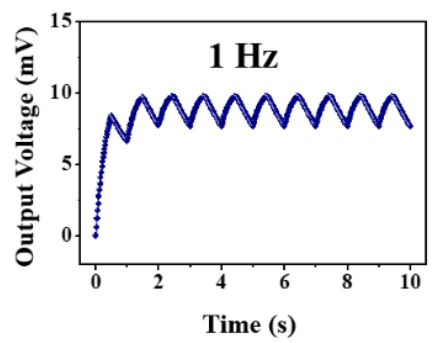

b

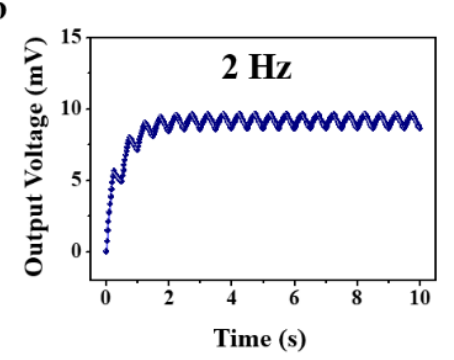

e

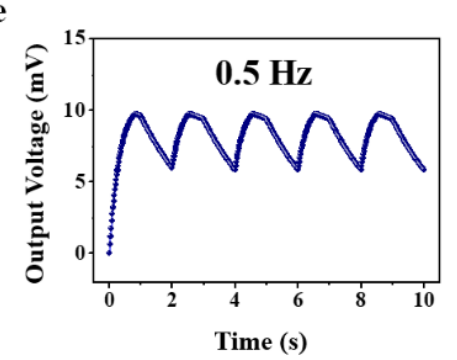

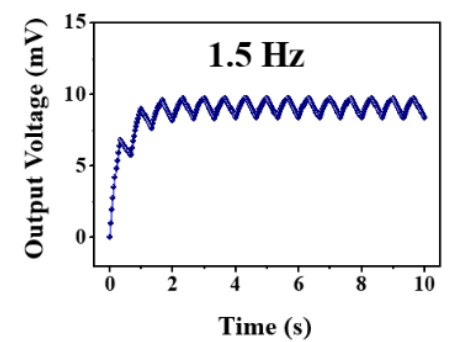

f

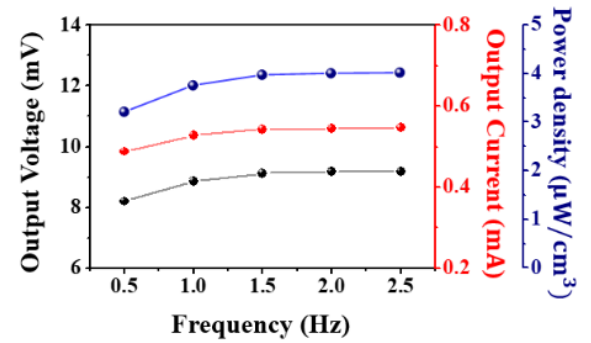

Figure S15. Output voltage signals for the HThTG with a tile spacing of $3.5 \mathbf{~ m m}$ when human touch temperature is repeatedly applied at (a) 2.5 , (b) 2 , (c) 1.5 , (d) 1 , and (e) $0.5 \mathrm{~Hz}$ at an external load of $16.8 \Omega$. (f) The analysis of output voltage, current, and power density according to frequency change. 
a

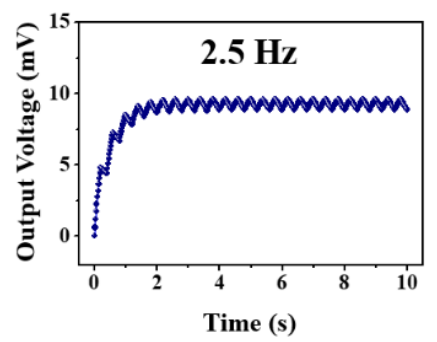

d

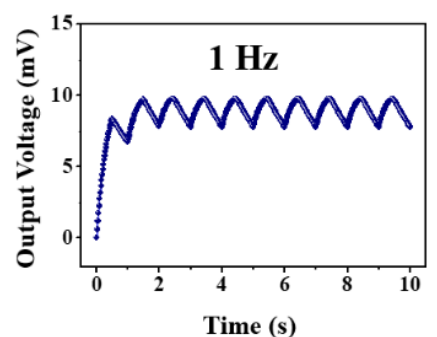

b

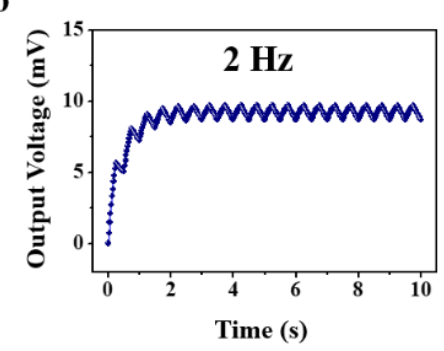

e

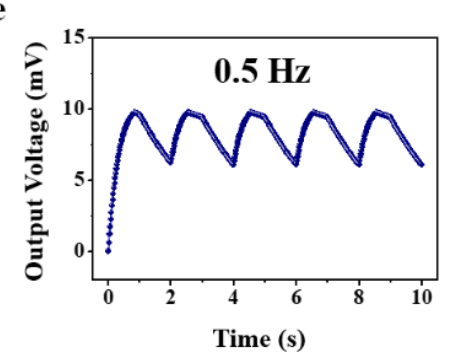

c

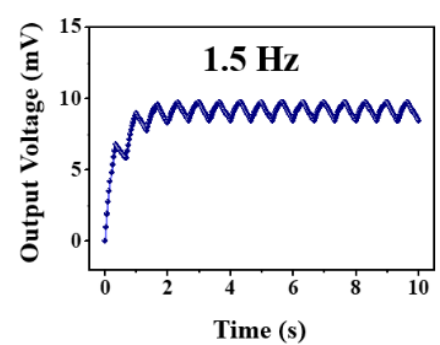

f

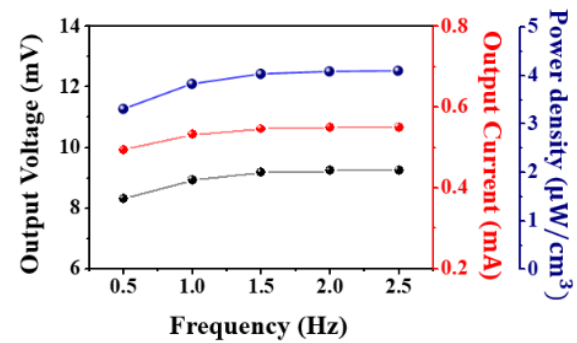

Figure S16. Output voltage signals for the HThTG with a tile spacing of $4 \mathbf{~ m m}$ when human touch temperature is repeatedly applied at (a) 2.5 , (b) 2, (c) 1.5 , (d) 1 , and (e) $0.5 \mathrm{~Hz}$ at an external load of $16.8 \Omega$. (f) The analysis of output voltage, current, and power density according to frequency change. 


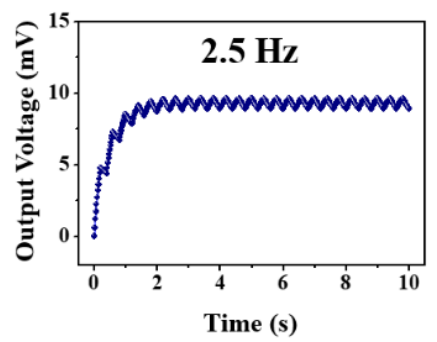

d

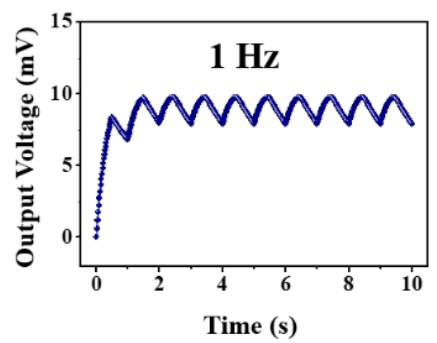

b

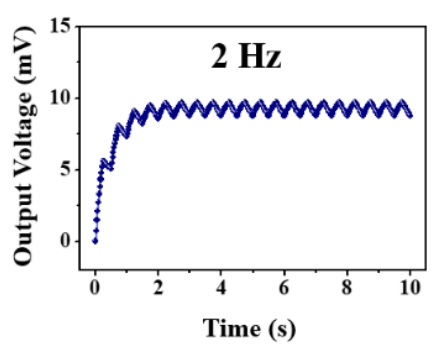

e

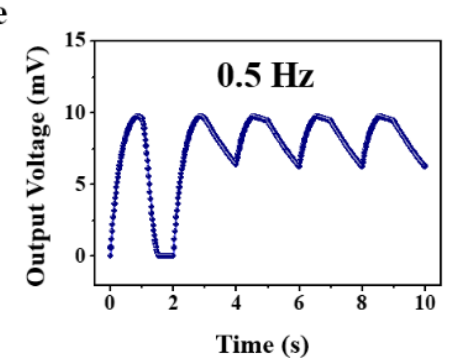

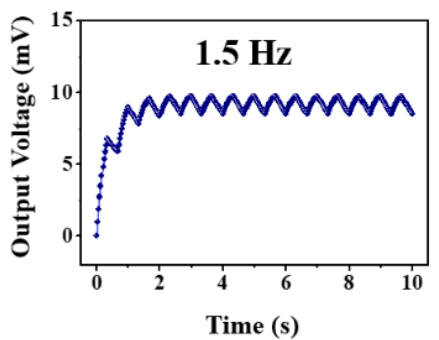

f

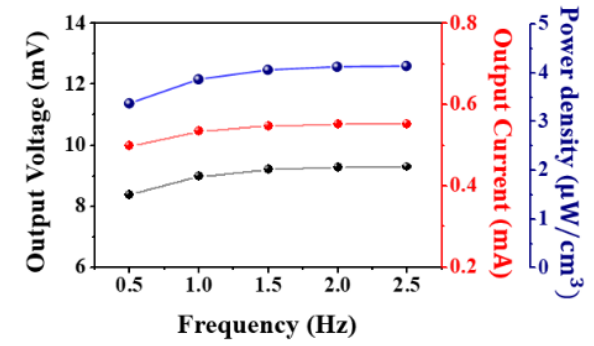

Figure S17. Output voltage signals for the HThTG with a tile spacing of $4.5 \mathrm{~mm}$ when human touch temperature is repeatedly applied at (a) 2.5, (b) 2, (c) 1.5 , (d) 1 , and (e) $0.5 \mathrm{~Hz}$ at an external load of $16.8 \Omega$. (f) The analysis of output voltage, current, and power density according to frequency change. 
a

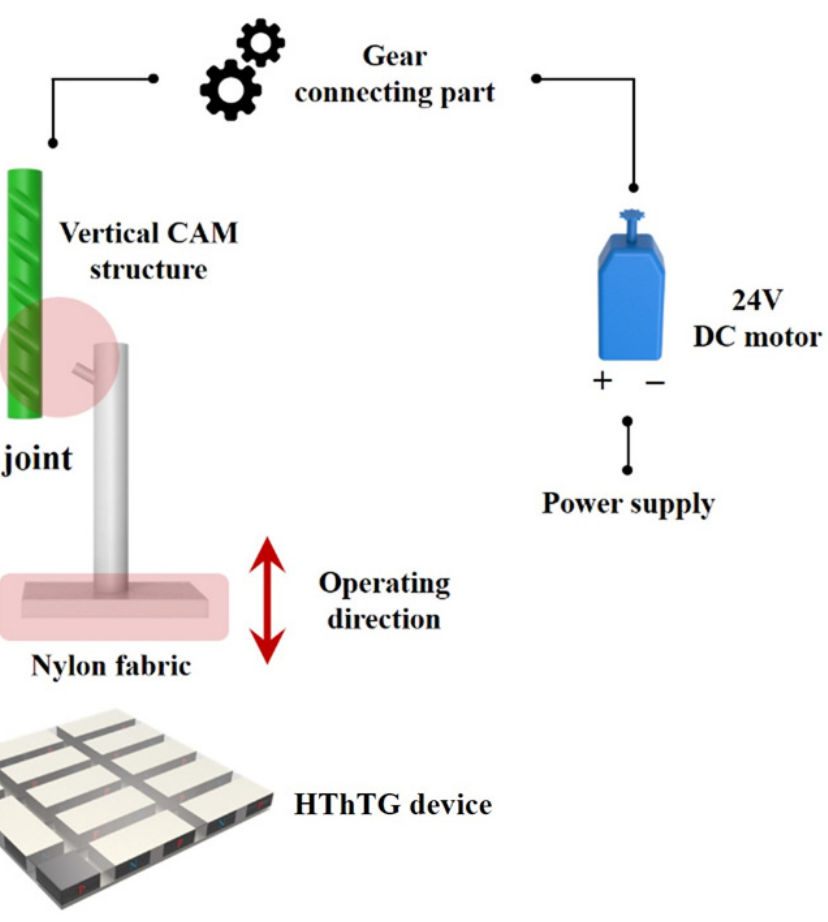

b

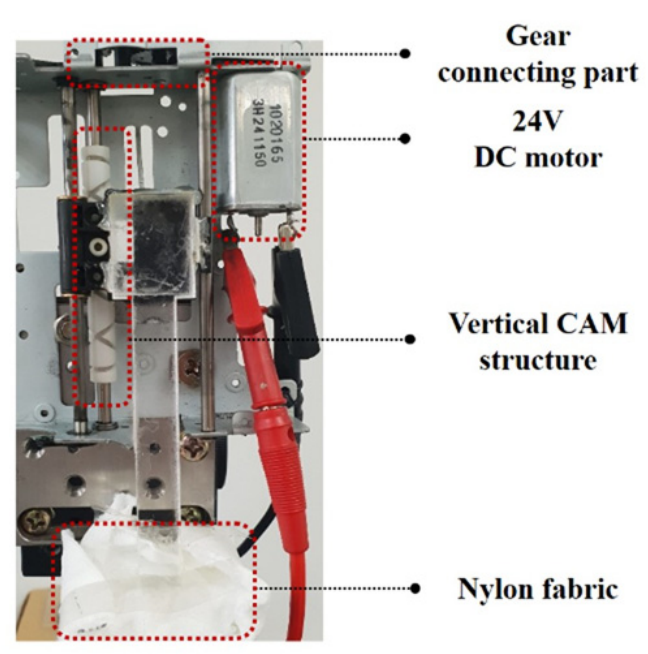

Figure S18. (a) A schematic representation and (b) an image of the homemade apparatus for triboelectric performance evaluation. 